\title{
Loss of contrast sensitivity following contusional eye injury
}

\author{
Y. CANAVAN AND D. B. ARCHER \\ From the Department of Ophthalmology, the Queen's University of Belfast, and the \\ Eye and Ear Clinic, Royal Victoria Hospital, Falls Road, Belfast
}

SUMMARY Contrast sensitivity was evaluated in 95 patients who regained a visual acuity of $6 / 6$ following a contusional injury to one eye. The injuries occurred 2 to 12 years prior to examination. A book of printed sinusoidal grating patterns of varying contrasts and spatial frequencies was used, and 15 patients were found to have a significant difference in contrast sensitivity between injured and uninjured eye. Eight patients had abnormalities of the media or fundus, and in 2 patients amblyopia was probably a factor. Five patients had a defect in contrast sensitivity in their injured eye, although no structural abnormalities could be detected on full clinical examination. Alterations in contrast sensitivity appeared to be a sensitive indicator of functional abnormality in eyes which appeared normal on ophthalmoscopy and other clinical investigations.

Contrast sensitivity is an important and measurable parameter of visual function, although its anatomical and physiological basis has yet to be fully explained. Loss of contrast sensitivity may occur in certain ocular disorders where there is no detectable alteration in visual acuity or field and where no structural abnormalities of the eye can be identified. Bodis-Wollner ${ }^{1}$ described loss of contrast sensitivity in patients with intracranial lesions and normal visual acuity, and Sjöstrand and Frisen ${ }^{2}$ noted significant loss of contrast sensitivity in patients with macular disease accompanied by only minimal loss of central visual functions. Patients with amblyopia $^{3}$ or myopia and astigmatism ${ }^{4}$ may also demonstrate loss of contrast sensitivity.

Arden, ${ }^{5}$ in 1978, described a simple technique for the clinical measurement of contrast sensitivity. He transferred a selection of 6 sinusoidal grating patterns of varying contrast and spatial frequency on to paper and produced a book which could be used rapidly and easily in the clinical evaluation of patients. The book was loose-leaf and each page was enclosed and protected by a plastic folder. Each text contained six sheets exhibiting sinusoidal grating patterns (plates 2-7), each sheet illustrating a grating of specific spatial frequency and designed to be read at $57 \mathrm{~cm}$. The contrast on each plate increased progressively from subthreshold (uniform

Correspondence to Professor D. B. Archer, Department of Ophthalmology, Royal Victoria Hospital, Belfast BT12 6BA. grey appearance) to higher and higher contrast areas. An arbitrary scale placed at the side of each plate facilitated the numerical recording of the patient's threshold position.

Arden and Gocukoglu ${ }^{6}$ reported on 57 patients with retrobulbar neuritis (with or without other signs of multiple sclerosis) and found that the grating test highlighted abnormalities in contrast sensitivity in affected as well as unaffected eyes. They found that the abnormalities in contrast sensitivity thresholds compared favourably with electrophysiological testing as a technique to evaluate abnormalities of visual functions in such patients. Arden and Jacobson ${ }^{7}$ determined that the grating test was also of value in screening patients for glaucoma. Patients with glaucoma gave higher test scores than the normal range of the population and their loss of contrast sensitivity corresponded to the severity of the disease. Patients with ocular hypertension also demonstrated abnormal contrast sensitivity, further enhancing the effectiveness of this test as a screening procedure for glaucoma.

This paper reports an investigation of contrast sensitivity in 95 patients who suffered a contusional injury to the eye but who regained a visual acuity of $6 / 6$ or better in the injured eye.

\section{Materials and methods}

In a recent survey ${ }^{8}$ of 1063 patients with contusional (nonperforating) eye injuries of sufficient severity to 
necessitate hospital admission it was found that 431 $(40.5 \%)$ regained a visual acuity of $6 / 6$ or better and were asymptomatic. Ninety-five of these patients were available for review, which was carried out between 2 and 12 years following injury. The patients' ages ranged from 10 to 75 years with an expected predominance in young adulthood.

Twenty-eight of the 95 patients were found to have no detectable structural abnormalities of the eyes on full ocular examination. Sixty-seven patients had significant structural alterations, of which the most common was recession of the anterior chamber angle. Fourteen patients had additional signs of anterior segment trauma including pupillary sphincter tears, iridodialyses, and localised lens opacities. Twelve patients were found to have lesions of the posterior segment of the eyes, the most common being retinal pigment epithelial alterations, small choroidal tears, or breaks in Bruch's membrane in the posterior and equatorial fundus.

Contrast sensitivity studies were carried out in this group of patients using the book of grating patterns and the technique advocated by Arden. ${ }^{5}$ Each eye was tested in turn, the other eye being covered. The injured and noninjured eyes were tested in a random fashion and appropriate reading spectacles used in those patients who required them. The patients were positioned at a table and the plates were scrutinised at a reading distance of $57 \mathrm{~cm}$ under indoor lighting conditions along with a near-by desk lamp ( $60 \mathrm{~W}$ bulb). The procedure was explained to the patient and one practice test was allowed. Each plate (2-7) was then read in turn, the plate being covered by a card which revealed only the subthreshold contrast, so that the patient appreciated only a uniform grey area. The card was then withdrawn slowly revealing higher and higher contrasts until the grating pattern became visible to the patient. The scale reading at this position was recorded, and the procedure was then repeated for each of the 6 plates and the scale readings for each plate indicating the contrast threshold summated to give a score.

The procedure was then repeated for the second eye. A difference of 10 or more between the scores for the 2 eyes was considered significant, indicating an abnormality of contrast sensitivity in the higher scoring eye.

\section{Results}

Of 95 patients reviewed 15 had significant loss of contrast sensitivity in the injured eye (i.e., a difference of more than 10 in the scores between the injured and uninjured eye). The score value difference between the 2 eyes ranged from 10 to $25+$
Table 1 Analysis of 15 patients with significant loss of contrast sensitivity in injured eye

\begin{tabular}{|c|c|c|c|}
\hline $\begin{array}{l}\text { Patient } \\
\text { number }\end{array}$ & $\begin{array}{l}\text { Distance vision } \\
\text { (with glasses if } \\
\text { habitually worn) }\end{array}$ & $\begin{array}{l}\text { Grating score } \\
\text { difference } \\
\text { between injured } \\
\text { uninjured eyes }\end{array}$ & $\begin{array}{l}\text { Clinical } \\
\text { examination of } \\
\text { the injured eye }\end{array}$ \\
\hline 1 & $6 / 6$ & $10 \cdot 0$ & Normal \\
\hline 2 & $6 / 6$ & $10 \cdot 5$ & Normal \\
\hline 3 & $6 / 6$ & $10 \cdot 5$ & Normal \\
\hline 4 & $6 / 6$ & $11 \cdot 5$ & Normal \\
\hline 5 & $6 / 6$ & $16 \cdot 0$ & Normal \\
\hline 6 & $6 / 6$ & $14 \cdot 5$ & ? Amblyopia \\
\hline 7 & $6 / 6$ & $16 \cdot 0$ & ? Amblyopia \\
\hline 8 & $6 / 6$ & $10 \cdot 0$ & Lens opacities \\
\hline 9 & $6 / 6$ & $10 \cdot 0$ & Choroidal tears \\
\hline 10 & $6 / 6$ & $10 \cdot 5$ & Lens opacities \\
\hline 11 & $6 / 6$ & $11 \cdot 0$ & $\begin{array}{l}\text { Sector optic } \\
\text { Atrophy }\end{array}$ \\
\hline 12 & $6 / 6$ & $11 \cdot 5$ & Choroidal tears \\
\hline 13 & $6 / 6$ & $24 \cdot 0$ & $\begin{array}{l}\text { Repaired retinal } \\
\text { Detachment }\end{array}$ \\
\hline 14 & $6 / 6$ & $24 \cdot 5$ & $\begin{array}{l}\text { Optic disc } \\
\text { Pallor }\end{array}$ \\
\hline 15 & $6 / 6$ & $25.0+$ & Choroidal tears \\
\hline
\end{tabular}

(Table 1). Patients 1 to 5 showed significant loss of contrast sensitivity in the injured eye, though they were asymptomatic and had a corrected visual acuity of $6 / 6$ or better. No physical abnormalities were detected on detailed ocular examination in this group to account for the discrepancy. Similar findings were noted in patients 6 and 7, but the interpretation of results was complicated, as patient 7 had a small intermittent divergent strabismus in his injured eye. The latter patient was unaware of the strabismus, and it was not possible to ascertain whether it predated the injury. Patient 6 also had a squint treated in childhood, but no significant deviation was noted on clinical evaluation.

Patients 8 to 15 were also found to have significant defects of contrast sensitivity in their injured eyes, but all patients in this group had structural abnormalities in the injured eyes which could have had an effect on contrast discrimination. Patients 8 and 10 had anterior and posterior cortical lens opacities respectively in the region of near-by iris tears or areas of iris atrophy. Three patients, 9, 12, and 15, had areas of retinal pigment epithelial atrophy or proliferation and areas of choroidal atrophy usually associated with 'choroidal tears'. In patient 9 only 
a small localised area of retinal pigment epithelial and choroidal atrophy was present (Figs. 1,2), but in cases 12 and 15 complex retinal pigment epithelial and choroidal degenerative changes were present in

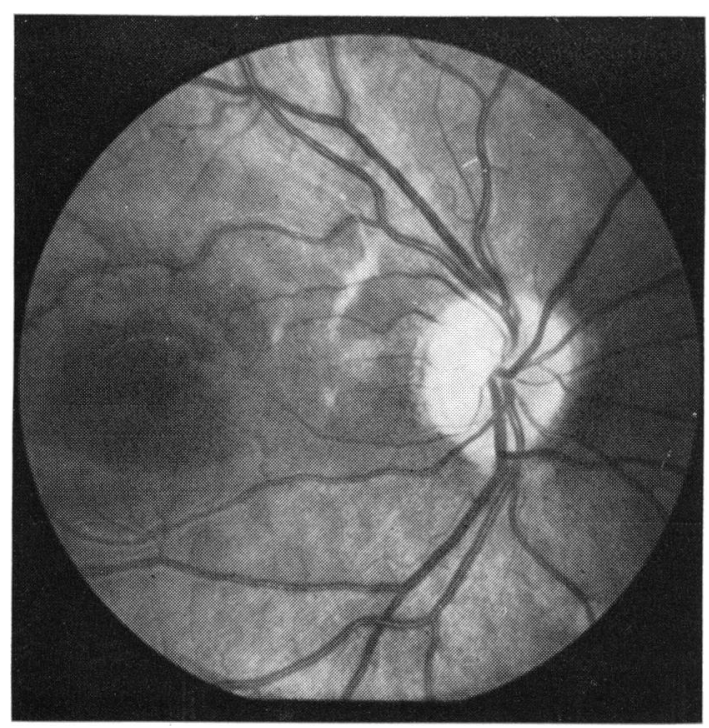

Fig. 1 Right fundus photograph of patient 9 showing 3 small areas of retinal pigment epithelial and choroidal atrophy involving the superior papillomacular region.

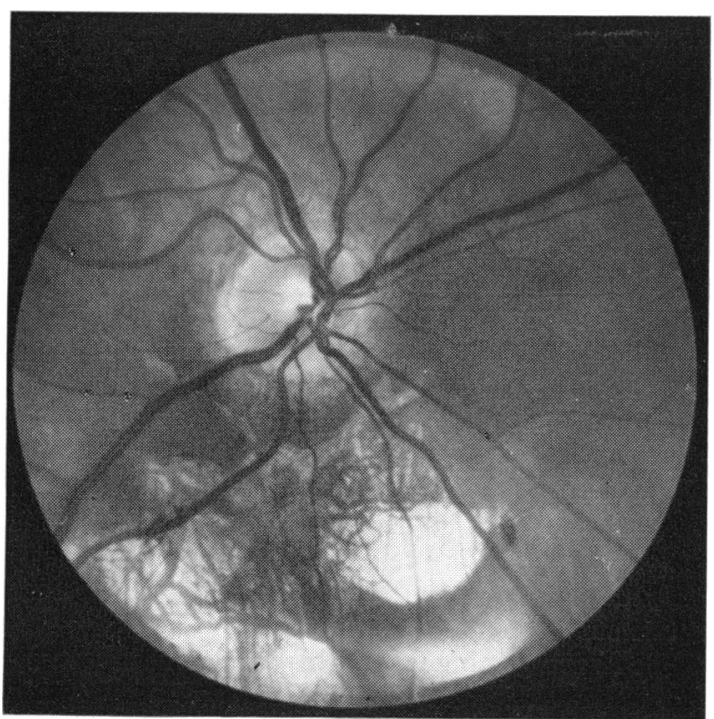

Fig. 3 Right fundus photograph of patient 12 demonstrating extensive patchy atrophy of the retinal pigment epithelium and choroid involving a sector inferior to the optic disc. the region of the posterior pole of the fundus (Figs. 3, 4). Patients 11 and 14 had significant optic atrophy. Patient 14 had moderate pallor of the optic disc, while in patient 11 the atrophic changes

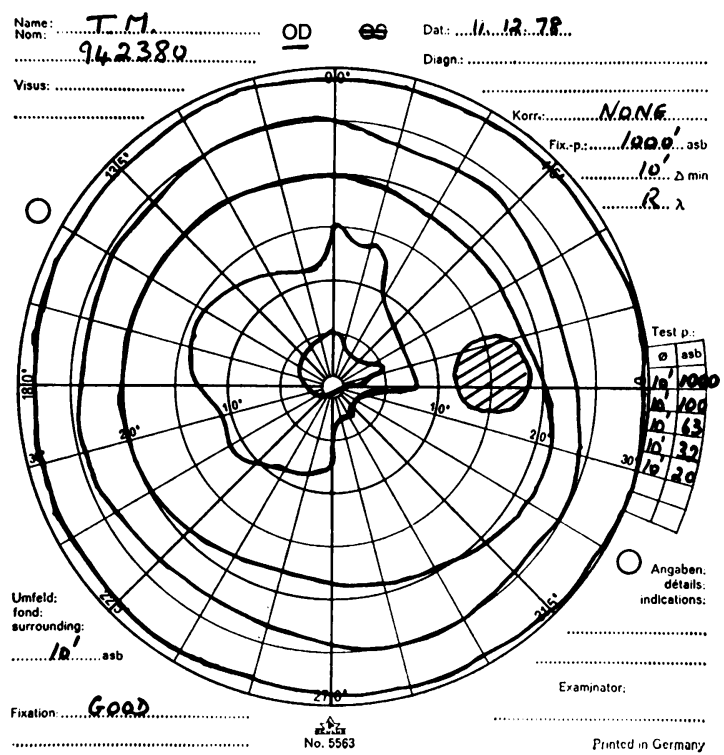

Fig. 2 Central visual field (Tübinger) of patient shown in Fig. 1 demonstrating inferotemporal constriction of the visual field in the $5^{\circ}$ and $10^{\circ}$ isoptres.

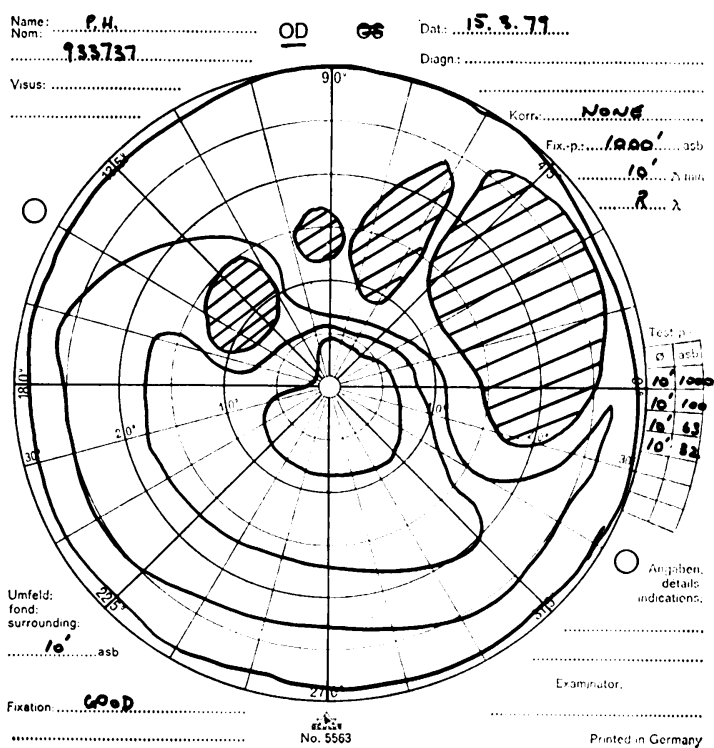

Fig. 4 Central visual field (Tübinger) of patient shown in Fig. 3. Several large scotomata involve the right superior field. The fovea is spared. 


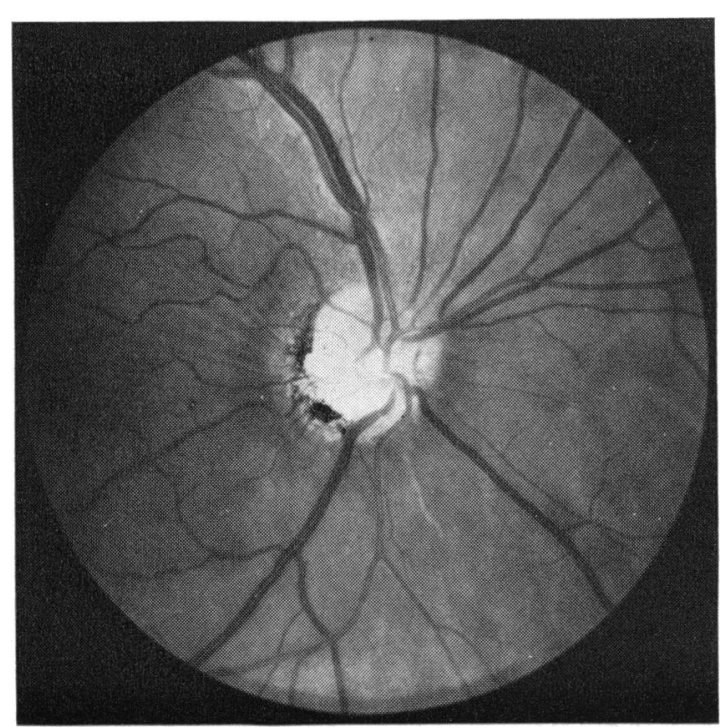

Fig. 5 Right fundus photograph of patient 11 demonstrating atrophy of the inferotemporal margin of the optic disc. Wrinkles in the internal limiting membrane radiate from the temporal part of the disc and nerve fibre striations are absent in the inferior retina.

were localised to an inferior sector of the right optic disc and associated with a superior altitudinal visual field defect (Figs. 5, 6).

Patient 13 developed a localised rhegmatogenous retinal detachment following injury and subsequently had an encirclement procedure to reattach the retina. This patient subsequently developed a diffuse retinal pigment epithelial disturbance over the superior half of the right fundus.

\section{Discussion}

Prolonged observation of a high-contrast grating reduces the visibility of a low-contrast grating of equal spatial frequency; however, sensitivity to gratings of other spatial frequencies remains unaffected..$^{910}$ Campbell and Robson ${ }^{11}$ suggested that within the nervous system there exist linearly operating, independent 'channels', selectively sensitive to limited ranges of spatial frequencies. Thus, one channel could be inactivated by adaptation while other functions remain unaffected. High spatial frequencies must be received at the fovea and the retinal periphery is probably receptive to lower frequencies. It is therefore likely that contrast sensitivity is affected in the presence of either peripheral or central retinal pathology. ${ }^{5}$ The ability to see low spatial frequency gratings is not limited by the refractive properties of the eyes. Difficulties in perceiving high frequency gratings, however, may

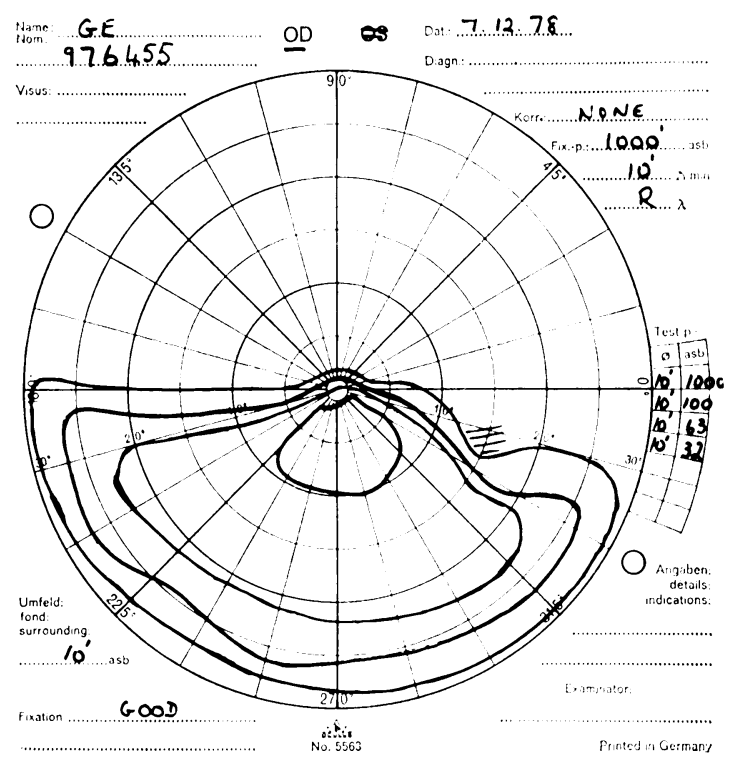

Fig. 6 Central visual field (Tübinger) of patient shown in Fig. 5. There is a superior altitudinal field defect, but the fovea is spared.

be attributable to a refractive error; therefore, difficulty with low frequency gratings identified patients suffering from a pathological condition. ${ }^{5}$ Until recently evaluation of contrast sensitivity has remained a laboratory investigation, most workers using oscilloscopic or televised displays. These methods were not suitable for clinical use, being expensive and difficult to transport. The development of a book of printed sinusoidal patterns has been a useful advance in testing contrast sensitivity in the clinical situation. The test is easily transported and rapidly performed. Patients in all age groups find the test relatively easy to understand, and a practice run using one plate is generally all that is required. Younger patients tend to see the grating pattern more quickly than older patients and hence have lower average scores. The test is therefore particularly useful in comparing right and left eyes of one patient and it is more difficult to compare results from patient to patient. There are certain variations in results depending on the observer's technique, and it is preferable that all tests in one study be carried out by one person, as was the case in this survey.

The Arden grating test in this particular investigation was reliable and reproducible, and only 2 false positive results occurred in 95 patients investigated. In 1 patient there was a marked and unexplained discrepancy in the scores between the 2 eyes, the uninjured eye having the higher score. A second 
patient who also had a significantly higher score in the uninjured eye gave a definite history of squint and amblyopia in this eye which could have accounted for the discrepancy.

This investigation has established that the majority of patients who regained a visual acuity of $6 / 6$ after contusional eye injury have no significant differences in contrast sensitivity in the injured and uninjured eyes. Fifteen patients, nevertheless, in this survey $(14 \%)$ showed a significantly raised score (greater than 10) in the injured eye on testing with the Arden gratings. Eight of these patients had well-defined structural abnormalities of either the mediae or fundi which undoubtedly contributed to the defect in contrast sensitivity. In general the more severe the structural abnormality of the eye the more marked was the difference in contrast sensitivity score between the normal and injured eyes. Injuries associated with impaired visual acuity showed correspondingly severe diminution in contrast sensitivity in the affected eye. ${ }^{8}$

It is significant that 2 patients with early anterior and posterior cortical lens opacities secondary to trauma also showed loss of contrast sensitivity in the affected eye. This observation has been made previously by $\operatorname{Arden}^{5}$ and explains why patients with early lens opacities of any aetiology may complain of visual disturbances which seem out of proportion to a visual acuity of $6 / 6$. In 2 further patients there were definite defects in contrast sensitivity in the injured eye where amblyopia was probably the contributory factor. Patients 1 to 5 are particulaly instructive as they showed grating score differences of 10 to 16 between the injured and uninjured eyes in the presence of a normal visual acuity and absence of symptoms. The only structural abnormality detected in these patients was a mild degree of anterior chamber angle recession which was not considered clinically significant. Contrast sensitivity testing therefore appears to be a sensitive indicator of functional abnormality in an eye judged to be normal using other clinical parameters.

We are grateful to the Eastern Area Health and Social Services Board for providing a research grant from the endowment fund of the Royal Victoria Hospital to facilitate this project.

\section{References}

1 Bodis-Wollner I. Visual acuity and contrast sensitivity in patients with cerebral lesions. Science 1972; 178: 769-71.

2 Sjöstrand J, Frisen L. Contrast sensitivity in macular disease. Acta Ophthalmol (Kbh) 1977; 55: 507-14.

3 Hess RF, Howell ER. The threshold contrast sensitivity function in strabismic amblyopia: evidence for a two type classification. Vision Res 1977; 17: 1049-55.

4 Fiorentini A, Maffei L. Spatial contrast sensitivity of myopic subjects. Vision Res $1976 ; 16$ : 437-8.

5 Arden GB. The importance of measuring contrast sensitivity in cases of visual disturbance. Br J Ophthalmol 1978; 62: 198-209.

6 Arden GB, Gocukoglu AG. A simple clinical test of contrast sensitivity demonstrates unsuspected visual losses in patients with minimal demyelinisation of the optic nerve. Arch Ophthalmol 1978; 96: 1626-9.

7 Arden GB, Jacobson J. A simple grating test for contrast sensitivity: preliminary results indicate value in screening for glaucoma. Invest Ophthalmol 1978; 17: 23-32.

8 Canavan Y. A ten year retrospective survey of eye injuries in Northern Ireland (1967-1976) with particular reference to blunt injury of the globe. Thesis submitted for the degree of Doctor of Medicine, Queen's University of Belfast, 1979.

9 Blakemore C, Campbell FW. Adaptation to spatial stimuli. J Physiol 1969; 200: 11P-13P.

10 Maffei L, Fiorentini A, Bisti S. Neural correlate of perceptual adaptation to gratings. Science 1973; 182: 1036-8.

11 Campbell FW, Robson JG. Application of Fourier analysis to the visibility of gratings. J Physiol 1968 197: $551-66$ 